\author{
Rianne Wittenberg \\ Joost F. Peters \\ Jeroen J. Sonnemans \\ Mathias Prokop \\ Cornelia M. Schaefer-Prokop
}

\section{Computer-assisted detection of pulmonary embolism: evaluation of pulmonary CT angiograms performed in an on-call setting}

Received: 25 May 2009

Revised: 12 August 2009

Accepted: 14 September 2009

Published online: 28 October 2009

(C) The Author(s) 2009.

This article is published with open access at Springerlink.com

R. Wittenberg $(\bowtie)$.

C. M. Schaefer-Prokop

Department of Radiology,

Academic Medical Centre,

Meibergdreef 9,

1105 AZ Amsterdam, The Netherlands

e-mail: r.wittenberg@amc.uva.nl

Tel.: +31-61-5370185

R. Wittenberg $\cdot$ M. Prokop

Department of Radiology,

University Medical Centre,

Heidelberglaan 100,

3508 GA Utrecht, The Netherlands

J. F. Peters · J. J. Sonnemans

Healthcare Informatics - Clinical

Science \& Advanced Development,

Philips Healthcare,

Veenpluis 4 ,

5684 PC Best, The Netherlands

\author{
C. M. Schaefer-Prokop \\ Department of Radiology, Meander \\ Medical Centre, \\ Utrechtseweg 160, \\ 3818 ES Amersfoort, The Netherlands
}

\begin{abstract}
Purpose: The purpose of the study was to assess the standalone performance of computerassisted detection (CAD) for evaluation of pulmonary $\mathrm{CT}$ angiograms (CTPA) performed in an on-call setting. Methods: In this institutional review board-approved study, we retrospectively included 292 consecutive CTPA performed during night shifts and weekends over a period of 16 months. Original reports were compared with a dedicated CAD system for pulmonary emboli (PE). A reference standard for the presence of PE was established using independent evaluation by two readers and consultation of a third experienced radiologist in discordant cases.
\end{abstract}

Results: Original reports had described 225 negative studies and 67 positive studies for PE. CAD found PE in seven patients originally reported as negative but identified by independent evaluation: emboli were located in segmental $(n=2)$ and subsegmental arteries $(n=5)$. The negative predictive value (NPV) of the CAD algorithm was $92 \%$ (44/48). On average there were 4.7 false positives (FP) per examination (median 2, range $0-42$ ). In $72 \%$ of studies $\leq 5 \mathrm{FP}$ were found, $13 \%$ of studies had $\geq 10$ FP. Conclusion: CAD identified small emboli originally missed under clinical conditions and found $93 \%$ of the isolated subsegmental emboli. On average there were 4.7 FP per examination.

Keywords Multidetector-row computed tomography - Computed tomography angiography $\cdot$ Pulmonary embolism · Computer-assisted diagnosis $\cdot$ Pulmonary arteries

\section{Introduction}

Acute pulmonary embolism (PE) is one of the most frequent potentially fatal diseases. Unfortunately, because of its unspecific clinical symptoms, pulmonary embolism is difficult to diagnose clinically. In two-thirds of the cases the diagnosis is missed if no additional diagnostic procedures are carried out [1-3].

The diagnostic imaging method of choice for the detection of PE in most institutions is contrast-enhanced pulmonary multidetector CT angiography (CTPA) [4-8]. However, each CTPA examination produces on average
300-500 axial images. Thin sections need to be reviewed because it is known that small emboli can be missed on thick sections [9]. Meticulous review of all CT slices is therefore time-consuming and requires a high level of attentiveness. The prevalence of PE in patients sent to CTPA is moderate to low, in the range of $10-35 \%$ according to current literature $[10,11]$. The chance of missing small emboli increases with time pressure and anatomical and technical complexity and decreases as readers become more experienced [12, 13].

Computer-aided detection (CAD) algorithms have been developed to help exclude pulmonary embolism and to 
improve the detection performance of observers. The purpose of this study was to assess the stand-alone performance of a computer-assisted detection (CAD) prototype on evaluation of pulmonary CT angiograms (CTPA) performed in an on-call setting. Performance of the CAD algorithm as a stand-alone system was compared with a reference standard and the original reports.

\section{Materials and methods}

\section{Patient selection}

In this institutional review board-approved study, we retrospectively included all 292 consecutive CTPA studies performed in a university hospital during night shifts and weekends over a period of 16 months between January 2007 and April 2008. All patients had been referred to the radiology department for CTPA because of suspected acute PE. Fourteen patients were excluded from further evaluation for the following two reasons: six CTPA examinations because of streak artefacts based on non-elevated arm positions and metallic material that made a diagnostic evaluation of the imaging impossible and eight examinations because the CAD algorithm did not work for these data sets. In two of these cases, the CAD algorithm failed because the patient had a pneumothorax on the left side or the left lung had been surgically removed. The other six patients had a trachea tube for respiratory ventilation resulting in a connection between extrathoracic air and intrapulmonary air and subsequent failure of the segmentation of the trachea and lungs.

The final study group therefore consisted of 278 patients: 138 male, 140 female, mean age 57 years (range 18-88). There were 133 inpatients (13 patients came from the intensive care unit), 5 patients from the outpatient clinic and 140 patients submitted to the emergency unit. In total, $23 \%$ studies were originally reported as positive for PE and $77 \%$ as negative.

\section{CT technique}

All CTPA examinations were acquired using 16- or 64-detector-row CT; 178 patients were examined by 16-detector-row CT (MX 8000 IXDT or Brilliance-16, Philips Medical Systems, Cleveland, OH) and 100 patients underwent 64-detector-row CT (Brilliance-64, Philips Medical Systems, Cleveland, OH). All CT images were acquired in a caudo-cranial direction from the level of the diaphragm to the lung apices within a single breath hold. A standard PE protocol was applied. The Stellant Dual CT Injector (Medrad Europe BV, Beek, The Netherlands) was used for intravenous bolus injection.

The 64-slice CTPA were obtained with $120 \mathrm{kV}$, $100 \mathrm{mAs}, 64 \times 0.625 \mathrm{~mm}$ collimation, rotation time $0.4 \mathrm{~s}$ and pitch 1.172. All images were reconstructed with $0.9-\mathrm{mm}$ slice thickness and $0.45-\mathrm{mm}$ overlap. Patients received $90 \mathrm{ml}$ of i.v. contrast medium (Ultravist 300, Schering, Berlin, Germany) injected at a flow rate of $5.0 \mathrm{ml} / \mathrm{s}$ and followed by a $30-\mathrm{ml} \mathrm{NaCl}$ chaser bolus. The 16-slice CTPA were obtained with $90 \mathrm{kV}, 180 \mathrm{mAs}, 16 \times$ $0.75 \mathrm{~mm}$ collimation, rotation time $0.5 \mathrm{~s}$, and pitch 1.188 . All images were reconstructed with $1.0-\mathrm{mm}$ slice thickness and $0.5-\mathrm{mm}$ overlap. Patients received $90 \mathrm{ml}$ of i.v. contrast medium (Ultravist 300), injected at a flow rate of $4.0 \mathrm{ml} / \mathrm{s}$ and followed by a $40-\mathrm{ml} \mathrm{NaCl}$ chaser bolus.

For both techniques, a bolus tracking method was applied with the ROI in the pulmonary trunk. The threshold to start data acquisition was set at $150 \mathrm{HU}$ and a start delay of $8 \mathrm{~s}$ after reaching the trigger threshold was used.

Original reports had been based on the evaluation of thin axial images. Multiplanar reconstructions and maximum intensity projections (MIP) were used at the discretion of the interpreting resident or radiologist.

\section{Data collection and analysis}

All examinations were analysed by CAD prototype software (Philips Healthcare, Best, The Netherlands). For the analysis of the CAD lesions, a reference standard was established by independent evaluation by two readers. A researcher specially trained in reading PE studies (R.W.) and an experienced chest radiologist $(>15$ year experience, C.S.P.) evaluated all datasets separately. In the case of discordant findings between these two readers, a third experienced chest radiologist ( $>15$ years experience, M.P.) was consulted. The readers were instructed to digitally mark each intravascular thrombus with regard to its anatomical location (e.g. pulmonary lobe) and the anatomical level of its proximal end (central, lobar, segmental and subsegmental). Standard nomenclature, derived from Boyden [14] and from Jackson and Huber [15], was used to identify the segmental and subsegmental structures. Care was taken that thrombi with continuous extension in various branching vessels were counted as a single lesion and that small subsegmental emboli were included.

In a second step, the CAD findings were compared with our reference standard and considered as true positive, false negative or false positive (Figs. 1, 2, 3). To define a CAD finding as true positive that had not primarily been seen by one of the readers, the presence of an intravascular contrast defect had to be securely confirmed by the other readers.

\section{Principles of the CAD algorithm}

CAD is a fully automated pulmonary emboli detection prototype system (Philips Healthcare, Best, The Netherlands). It starts from automatic segmentation of the trachea and lungs. The resulting lung volume after morphological processing 


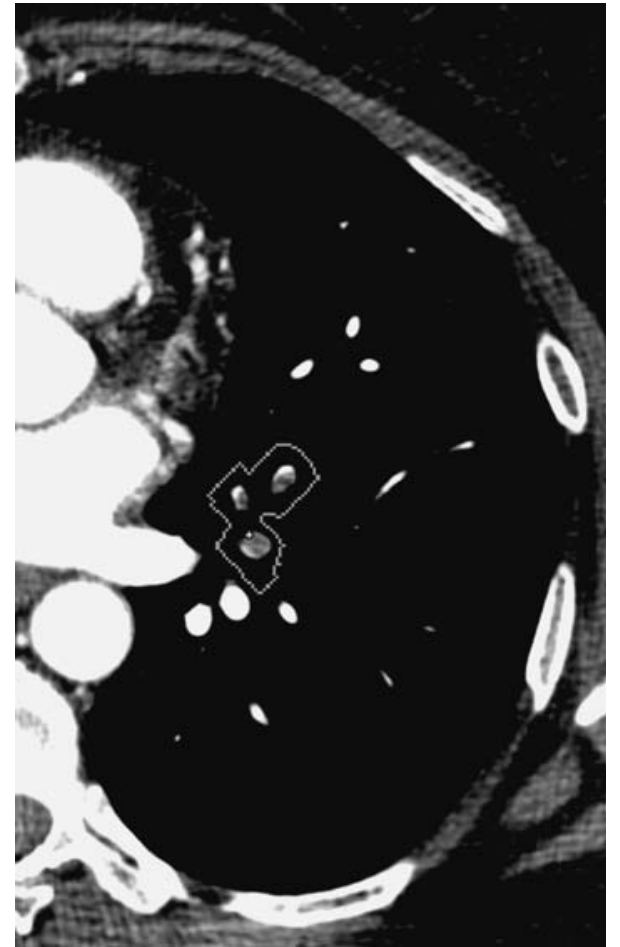

Fig. 1 Classis embolus found by CAD

contains both tissue and air. Subsequently, pulmonary vessels are segmented within this volume, starting with segmentation of all structures above $-100 \mathrm{HU}$. Second, a vessel-tracking algorithm is applied to extract the pulmonary vessels [16]. After pruning the vessels, vessel cross-sectional images perpendicular to the centrelines are computed. Grey value analysis of these cross-sectional images is applied to find candidate locations for pulmonary embolism. No pulmonary emboli are found outside the lung segmentation. Features for identifying pulmonary embolism include stretches of vessels that are completely occluded as well as areas that contain contrast-to-tissue transitions. Candidate lesions that the CAD identifies as potential PE are clustered and presented to the reader, indicated by an ROI around the affected vessel. The processing requires about $30 \mathrm{~s}$ per examination and is carried out automatically in the background during initial evaluation by the radiologists. Candidate lesions are then shown only on demand.

\section{Statistical analysis}

Statistical analysis was performed with SPSS (version 15.0 statistics UK). Sensitivity for the detection of PE by CAD was assessed on a per-patient as well as on a per-lesion basis, the latter with respect to the main, lobar, segmental and subsegmental levels. Specificity and the negative predictive value (NPV) of an examination without any candidate lesions found by the CAD algorithm were calculated.

\section{Results}

Distribution of emboli and sensitivity per embolus

Our reference standard determined that 68 out of 278 patients were positive for PE. A total of 377 emboli were detected in the 68 patients. Twenty-three emboli were localised in the main pulmonary arteries, 41 in lobar, 80 in segmental and 233 in the subsegmental arteries. In 37 patients, there were emboli extending into the main and/or lobar arteries. Thirty-one patients had only segmental and/ or subsegmental emboli. Fourteen patients had isolated subsegmental emboli. The per-embolus sensitivity of the $\mathrm{CAD}$ as a stand-alone system was $87,78,79$ and $61 \%$ respectively for emboli in the main, lobar, segmental and subsegmental arteries.

\section{Patient-based evaluation}

CAD correctly identified PE in seven patients originally reported as negative. In five patients, CAD found a solitary embolus in a segmental $(n=2)$ or subsegmental $(n=3)$ artery. In two patients, CAD found two subsegmental emboli.

On a per-patient basis, the sensitivity of the CAD system was $94 \%(64 / 68)$ and the specificity $21 \%(44 / 210)$. CAD

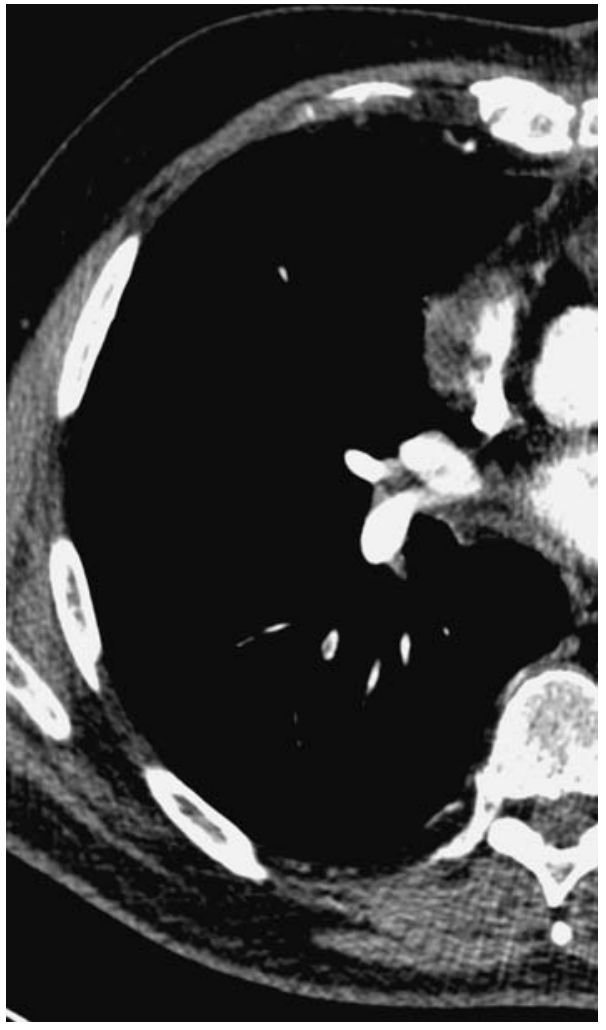

Fig. 2 Subsegmental embolus missed by primary readers 


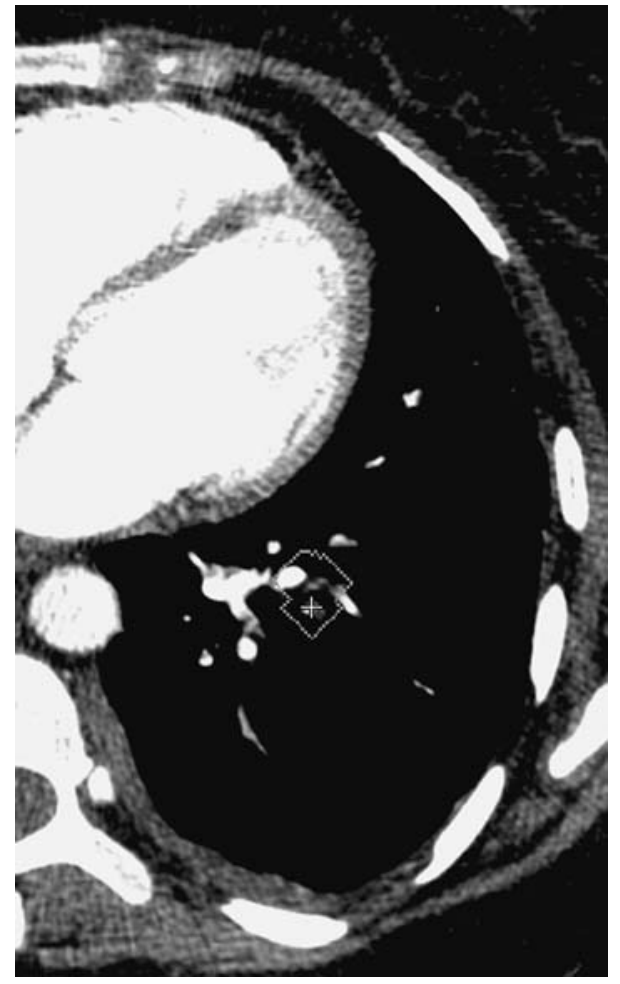

Fig. 3 False-positive candidate lesion found by CAD due to motion artifacts

correctly identified 93\% (13/14) of the patients with isolated subsegmental emboli.

In four patients, we found no embolism although the original reports had been positive. In $21 \%$ of the negative cases, there were no FP findings by CAD. The negative predictive value (NPV) of an examination without any candidate lesion found by the CAD algorithm was $92 \%$ (Tables 1 and 2).

\section{Analysis of false positives}

For the remaining 278 examinations, the CAD algorithm showed on average 4.7 FP lesions per examination (median 2 , range $0-42$ ) with most lesions located in non-arterial structures such as veins $(30 \%)$ or intrapulmonary opacifications $(22 \%)$. There were on average $3.5 \mathrm{FP}$ lesions (median 2.5, range 0-15) in the group of patients with PE

Table 1 Diagnosis according to the original reports versus the reference standard

\begin{tabular}{llll}
\hline & $\begin{array}{l}\text { Standard } \\
\text { positive }\end{array}$ & $\begin{array}{l}\text { Standard } \\
\text { negative }\end{array}$ & Total \\
\hline Original report positive & 61 & 4 & 65 \\
Original report negative & 7 & 206 & 213 \\
Total & 68 & 210 & 278 \\
\hline
\end{tabular}

Table 2 Diagnosis based on the CAD findings compared with the reference standard

\begin{tabular}{llll}
\hline & $\begin{array}{l}\text { Standard } \\
\text { positive }\end{array}$ & $\begin{array}{l}\text { Standard } \\
\text { negative }\end{array}$ & Total \\
\hline CAD positive & 64 & 166 & 230 \\
CAD negative & 4 & 44 & 48 \\
Total & 68 & 210 & 278 \\
\hline
\end{tabular}

and 5.0 (median 2, range 0-42) in the group without PE. In $72 \%$ of the examinations CAD indicated $\leq 5 \mathrm{FP}$, in $13 \%$ of the examinations CAD indicated $\geq 10 \mathrm{FP}$. Nine examinations had more than $20 \mathrm{FP}$ lesions, and all of them were negative for the presence of PE according to the standard.

\section{Discussion}

The arterial pulmonary vasculature is a complex anatomical structure whose detailed analysis requires a structured reading approach for the detection of emboli. Thorough analysis of the pulmonary vasculature requires scrolling through the 300-500 axial CT sections multiple times, a time-consuming technique which requires continuous concentration.

The purpose of this study was to assess the performance of this CAD prototype and get an estimation of its potential diagnostic impact. We tested CAD as a stand-alone system. We chose to analyse CTPA examinations that had been performed during night shifts and weekends because they are often read by less experienced colleagues or under time pressure. For these reasons, these examinations may especially benefit from the availability of a CAD system. We compared the CAD results with our reference standard and the original reports.

Our results demonstrate that CAD picked up segmental and subsegmental emboli that were confirmed by the reference standard in seven patients who had been described not to have a PE in the original report. In two patients a solitary embolus had been missed in a segmental artery, in three patients a solitary subsegmental embolus was missed and in two patients two subsegmental emboli were missed.

Our results are compatible with previously published results from smaller studies that both included reader performance. In a study by Engelke et al., 56 CTPA were evaluated by two experienced and two inexperienced readers with CAD as a second reader [17]. They showed the benefit of CAD for the detection of emboli at the segmental and subsegmental levels for the less experienced readers. Das et al. came to the same conclusions [18]: at the subsegmental level the sensitivity of three readers (with 1 and 6 years of experience) increased with CAD from 80 to $92 \%$, from 82 to $90 \%$ and from 63 to $81 \%$. However they only selected good quality images (vessel enhancement 
$>200 \mathrm{HU}$ ) and excluded patients with underlying lung diseases. In both studies statistics were carried out only on a per-lesion basis.

It is important to note that in our study, we consecutively included all off-hour studies obtained within a certain time period. Thus we also explicitly included images of limited quality, e.g. on the basis of suboptimal enhancement or breathing artefacts. The study group also included images showing underlying lung disease, such as infiltrates or atelectases, another factor that might negatively influence the performance of a CAD system. In total we had to exclude 14 examinations: 8 because the CAD algorithm did not work for these data sets and 6 scans that were considered as non-diagnostic on the basis of massive streak artefacts.

Detection of emboli by CAD has been shown to depend on the level of the embolus (segmental or subsegmental) and the degree of obstruction. The study by Zhou et al. used 14 positive patients to test their CAD algorithm. Their sensitivity for partially (20-80\%) occluded arteries was $84 \%$ for emboli in central, lobar and segmental arteries and dropped to $64 \%$ for emboli in subsegmental arteries [19]. If the vessel was minimally obstructed $(\leq 20 \%)$, sensitivity dropped to 65 and $33 \%$ respectively. The sensitivity decreased further to 59 and $27 \%$ respectively with an obstruction of $\geq 80 \%$.

We included all obstruction levels and found the sensitivity of CAD as a stand-alone system to be $87 \%$ for the central, $78 \%$ for the lobar, $79 \%$ for the segmental and $61 \%$ for the subsegmental emboli.

On a per-patient basis, sensitivity of the CAD was $94 \%$. Despite the variable image quality within the study group and the presence of underlying lung disease (in $n=200$ ), CAD was able to identify 13 out of 14 patients with isolated subsegmental emboli. This emphasises the potential added value of the system for the detection of small emboli, a task for which human observers are also known to have reduced sensitivity. The clinical importance of isolated subsegmental emboli is still uncertain: it is suggested that isolated small emboli may be an indicator of deep venous thrombosis and therefore predictor of more severe embolic events in the future. Furthermore it was stated that the clinical relevance of small peripheral emboli is larger in individuals with cardiopulmonary restriction [8].

A large proportion $(n=62 / 90,69 \%)$ of subsegmental emboli in our study that were missed by CAD were present in patients with central or lobar emboli. For these cases of central emboli, the detection of additional subsegmental emboli by CAD would have played a negligible clinical role unless the total embolus burden were used to direct patient management. Some subsegmental emboli $(n=$ $12 / 90,13 \%$ ) were missed because they were smaller than $2 \mathrm{~mm}$, which is below the threshold of the CAD algorithm, or because they had led to complete obstruction with a sudden cessation of the subsegmental arteries, which was not detected by CAD.
CAD missed 4 patients out of 68 positive patients with in total one lobar embolus, four segmental emboli and one subsegmental embolus. These four false-negative examinations were not characterised by especially low vascular enhancement or overlying motion artefacts. From a technical point of view, we were not able to identify obvious reasons why CAD was not able to identify the emboli in these four patients.

Most CTPA examinations done in clinical routine are eventually negative for the presence of PE. We found a negative predictive value (NPV) of $92 \%$ for examinations in which CAD did not find any candidate lesion. While an NPV of $92 \%$ is not yet sufficient for complete reliance on a negative CAD reading, it underlines the potential role of CAD as a second reading to reassure a reader when excluding PE. On the other hand, we have to state that only 44 out of the 210 negative cases did indeed have a completely negative $\mathrm{CAD}$ reading. Most negative $\mathrm{PE}$ examinations show differing numbers of false-positive CAD lesions. The specificity of the algorithm on a perpatient basis is very low at $21 \%$ indicating that there is still a need for considerable improvement and that readers have to learn to efficiently rule out false-positive candidate lesions if the application of CAD is to be beneficial.

Our results are comparable to those of the study by Walsham et al. [20]. They found an NPV of $84 \%$ and a specificity of $20 \%$ in a smaller $(n=100)$, but also nonselected patient group. While Zhou et al. showed an average of 14.4 false positives per study, our system produced 4.7 false positives per study. Under the assumption that a maximum of 5 false positive lesions per examination marked by CAD represents an acceptable level in clinical routine, this criterion was met in $72 \%$ of our patients. Thus most examinations produce a relatively low number of false-positive lesions. The median number of false-positive lesions was 2 with most located in nonarterial structures such as veins $(30 \%)$ or intrapulmonary opacifications $(22 \%)$. Thus it is likely that in most scans the false-positive lesions can easily be sorted out. The exact impact of the false-positive lesions on diagnostic accuracy and reading time, however, remains to be determined in a reader study. More than 10 false-positive lesions were seen in $13 \%$ of the CTPA examinations; not surprisingly, higher numbers of false-positive lesions correlated with lower image quality. Further reduction of false-positive lesions per image is needed as too many may have a negative influence on diagnostic performance, lead to an unnecessarily prolonged reading time and might even cause unnecessary treatment.

Our study suffers from some limitations. There is no absolute reference standard for CTPA studies. Diagnostic pulmonary catheter angiography has been completely substituted by multidetector CTPA and had additionally been questioned as reference standard anyway. We therefore established a reference standard by independent evaluation 
of two readers with a third reader in case of discordant findings.

We evaluated the stand-alone performance of the CAD software to provide an estimate of the performance of the algorithm. In our study group, $10 \%$ of PE patients were originally missed by the on-call radiologist, all of whom had PE identified by CAD. However, comparison between the performance of CAD as a stand-alone system and that of the original reports is not sufficient to define the role of CAD in a clinical setting. The potential benefit of a CAD system strongly depends on the interaction with the reader, his or her experience and ability to discriminate between true- and false-positive candidate lesions. More studies are necessary to evaluate the use of CAD as a second reading and to assess its impact on the reader's capability not only to detect emboli but also to exclude pulmonary embolism.
In summary, CAD can identify segmental and subsegmental pulmonary emboli that are missed by on-call radiologists, with an average of 4.7 false-positive lesions per examination. A CTPA without any CAD candidates has a high negative predictive value, which may serve as reassurance for less experienced readers. Furthermore CAD found most of the isolated subsegmental emboli, which are clinically the most difficult emboli to find.

Acknowledgements R. Wittenberg receives a research grant from Philips Medical Systems. J.F. Peters and J.J. Sonnemans are employees of Philips Medical Systems.

Open Access This article is distributed under the terms of the Creative Commons Attribution Noncommercial License which permits any noncommercial use, distribution, and reproduction in any medium, provided the original author(s) and source are credited.

\section{References}

1. British Thoracic Society (2003) British Thoracic Society guidelines for the management of suspected acute pulmonary embolism. Thorax 58:470-483

2. Olin JW (2002) Pulmonary embolism. Rev Cardiovasc Med 3(Suppl 2):S68 S75

3. Tapson VF (2008) Acute pulmonary embolism. N Engl J Med 358:10371052

4. Coche E, Pawlak S, Dechambre S et al (2003) Peripheral pulmonary arteries: identification at multi-slice spiral CT with $3 \mathrm{D}$ reconstruction. Eur Radiol 13:815-822

5. Ghaye B, Szapiro D, Mastora I et al (2001) Peripheral pulmonary arteries: how far in the lung does multi-detector row spiral CT allow analysis? Radiology 219:629-636

6. Remy-Jardin M, Remy J, Artaud D et al (1997) Peripheral pulmonary arteries: optimization of the spiral CT acquisition protocol. Radiology 204:157-163

7. Remy-Jardin M, Tillie-Leblond I, Szapiro D et al (2002) CT angiography of pulmonary embolism in patients with underlying respiratory disease: impact of multislice CT on image quality and negative predictive value. Eur Radiol 12:1971-1978
8. Schoepf UJ, Costello P (2004) CT angiography for diagnosis of pulmonary embolism: state of the art. Radiology 230:329-337

9. Schoepf UJ, Holzknecht N, Helmberger TK et al (2002) Subsegmental pulmonary emboli: improved detection with thin-collimation multi-detector row spiral CT. Radiology 222:483-490

10. Torbicki A, Perrier A, Konstantinides S et al (2008) Guidelines on the diagnosis and management of acute pulmonary embolism: the task force for the diagnosis and management of acute pulmonary embolism of the European Society of Cardiology (ESC). Eur Heart J 29:2276-2315

11. van Belle A, Buller HR, Huisman MV et al (2006) Effectiveness of managing suspected pulmonary embolism using an algorithm combining clinical probability, D-dimer testing, and computed tomography. JAMA 295:172-179

12. Chan HP, Hadjiiski L, Zhou C et al (2008) Computer-aided diagnosis of lung cancer and pulmonary embolism in computed tomography - a review. Acad Radiol 15:535-555

13. Schaefer-Prokop C, Prokop M (2005) MDCT for the diagnosis of acute pulmonary embolism. Eur Radiol 15 (Suppl 4):D37-D41

14. Boyden E (1955) Segmental anatomy of the lungs. McGraw-Hill, New York
15. Jackson C, Huber J (1943) Correlated applied anatomy of the bronchial tree and lungs with a system of nomenclature. Dis Chest 9:319-326

16. Buelow T, Wiemker R, Blaffert $\mathrm{T}$ et al (2005) Automatic extraction of the pulmonary artery tree from multi-slice CT data. Proc SPIE 5746:730-740

17. Engelke C, Schmidt S, Bakai A et al (2008) Computer-assisted detection of pulmonary embolism: performance evaluation in consensus with experienced and inexperienced chest radiologists. Eur Radiol 18:298-307

18. Das M, Muhlenbruch G, Helm A et al (2008) Computer-aided detection of pulmonary embolism: influence on radiologists' detection performance with respect to vessel segments. Eur Radiol 18:1350-1355

19. Zhou C, Chan HP, Patel S et al (2005) Preliminary investigation of computeraided detection of pulmonary embolism in three-dimensional computed tomography pulmonary angiography images. Acad Radiol 12:782-792

20. Walsham AC, Roberts HC, Kashani HM et al (2008) The use of computeraided detection for the assessment of pulmonary arterial filling defects at computed tomographic angiography. J Comput Assist Tomogr 32:913-918 